\title{
Patrones de conducta de los usuarios de un Servicio de Urgencia adosado a Hospital Tipo 4
}

CÉSAR GALAZ(1), RODRIGO GODOY(1), JAVIER CISTERNAS(1), GLADYS YENTZEN(2), MARÍA DE LA FUENTE(2)

\section{RESUMEN}

Estudio realizado en el Hospital de Llay Llay para determinar relación entre percepción usuaria de urgencia y atención médica recibida. Se describen las características de los usuarios del Servicio de Urgencia y se realiza una encuesta. Se definió: Percepción Usuaria: Urgente y No Urgente, Atención Médica Urgente y No Urgente, Usuario Infrecuente, Frecuente y Policonsultante. La población presenta niveles de consulta similares en todo el año, no observándose alzas que diferencien períodos de mayor consulta; además existe preferencia de consulta el lines, con disminución los sábado y domingo. De los 104 encuestados de este estudio, una mayoría correspondió a mujeres. El promedio de edad fue de 42 años sin diferencias entre sexos. Se encontró una coincidencia en la preferencia de atención y el lugar en donde se contestó la encuesta. Los motivos por los cuales prefieren el Servicio de Urgencia incluyen: la resolución rápida, la no solicitud de hora previa y la realización de exámenes en forma inmediata. Los motivos para elegir el Consultorio incluyen: entrega gratuita de fármacos y conformidad con la atención. El tiempo de espera no sería un factor que influye en la elección. Se demuestra, la existencia de factores no relacionados con la necesidad de atención inmediata: un 28,6\% de los hombres y un 34,4\% de las mujeres acudió al Servicio de Urgencia con una Percepción Usuaria No Urgente. Entre las opciones No Urgentes propuestas, destacan la falta de disponibilidad de horas en el Consultorio y la utilización del Servicio de Urgencia sólo por preferencia personal. Al evaluar la capacidad discriminativa entre Urgente y No Urgente la sensibilidad es 78\%, la especificidad es $55 \%$, los Falsos Positivos un $17 \%$ y Falsos Negativos 52\%. El porcentaje de Pacientes frecuentes es alrededor de $50 \%$ con 1 in $6,1 \%$ de Policonsultantes y un 14\% de Hospitalización. Se concluye que existirian patrones de conducta de los usuarios, predecibles y cuantificables que permitirian la implementación de medidas que incentiven el buen uso de los Servicios de Urgencia.

Palabras clave: Percepción, Servicios de Urgencia, conducta, encuesta, hospital tipo 4.

\section{ABSTRACT}

PATTERNS OF BEHAVIOR OF USERS OF AN EMERGENCY SERVICE ADDORSED TO A TYPE 4 HOSPITAL

Study carried out in the Llay Llay Hospital to determine the relation between user perception of emergency and medical care provided. Characteristics of the Emergency Service users are

(1) Interno de Medicina. Facultad de Medicina. Universidad de Chile. antonin gt@hotmail.com

(2) Escuela de Salud Páblica. Facultad de Medicina. Universidad de Chile. 
described and a personal interview is carried out. The following were defined: User Perception: Urgent and Non-urgent; Urgent and Non-urgent Medical Attention; Infrequent or Frequent User or Polyconsultant. The population presents similar consultancy levels throughout the year, no rises being observed pointing to higher consultancy periods; also, there exists a preference for consulting on Mondays, with a slackening toward Saturdays and Sundays. Of the 104 persons interviewed for this study, the majority were women. Average age was 42 years, there being no difference between sexes. There was coincidence both in care preference and where the interview took place. Reasons for preferring the Emergency Service include: rapid resolution, previous appointment not required, and immediate taking of exams. Reasons for choosing the Clinic include: free delivery of pharmaceuticals and conformity with medical care received. Waiting time was not considered a factor having an influence on the choice. The existence of factors not related to a need for immediate care was proved: $28.6 \%$ of males and $34.4 \%$ of females attended the Emergency Service with a "non-urgent user perception". Among the "non-urgent" options suggested, lack of availability of outpatient hours in the Clinic and use of the Emergency Service only due to personal preference, stand out. Upon evaluating the discriminative capacity between "urgent" and "non-urgent", sensitivity is $78 \%$ and specificity is $55 \%$, the "false positive" are $17 \%$ and the "false negative", 52\%. The percentage of "frequent patients" is around $50 \%$ with a $6.1 \%$ of "polyconsultants" and a $14 \%$ of "hospitalizations". The conclusion is that predictable and quantifiable user patterns of behavior would exist that would allow for the implementation of measures that would induce a good use of Emergency Services.

Key words: Perception, Emergency Services, behavior, interview, hospital type 4.

\section{INTRODUCCIÓN}

Los Servicios de Urgencia (SU) ocupan un lugar prioritario en el ámbito de salud pública, tanto por la cantidad de pacientes que atienden, como por la relevancia de los servicios que imparten. La OMS define Urgencia como "la aparición fortuita por cualquier causa o actividad de un problema de gravedad variable que genera la conciencia de una necesidad inminente de atención, por parte del sujeto que lo sufre o su familia". Esto trae implícita la capacidad del usuario de distinguir lo que es o no es urgente, lo que denominamos Percepción Usuaria de Urgencia. En el personal de salud de los Servicios de Urgencia existe la creencia que la mayoría de las consultas realizadas no requieren una atención inmediata, no existiendo necesariamente correlación entre la percepción usuaria y potencial riesgo para la salud del pacientel.2. Estudios previos lo avalan, siendo los pacientes que requieren una atención compleja y urgente, entre 1 y $3,5 \%$. Estudios extranjeros muestran cifras de hasta un $25 \% 5,6$. Esto agrava problemas ya existentes, como sobredemanda de atención, aumento de tiempos de espera y disconformidad con la atención otorgada y aumentos en los costos de la salud ${ }^{7-9}$, lo que es relevante al momento de atender gran cantidad de público sin un sistema que diferencie a los pacientes urgentes de los no urgentes.

Este estudio fue realizado en Llay Llay, $\mathrm{V}$ Región, que cuenta con 21.644 habitantes, 15.867 de ellos mayores de 15 años.

El Hospital San Francisco de Llay Llay, es un Hospital tipo 4 con servicio de Policlínico y Urgencia adosados, lo que permite al usuario elegir el lugar de atención, según su criterio o percepción de urgencia, eliminando factores como la accesibilidad al servicio.

Existen factores distintos a la Percepción de Urgencia que determinan la elección de un servicio u otro por parte del usuario ${ }^{10}$. Esto se vería reflejado en una baja relación entre la percepción del usuario y la atención médica recibida, lo que diferenciaría a grupos poblacionales que preferirían la atención de Urgencia per se $\mathrm{e}^{11}$.

Se plantean las siguientes hipótesis:

Lo que motiva al individuo a consultar en un Servicio de Urgencia no es sólo la percepción de urgencia del problema sino otros factores subsidiarios como rapidez de 
la atención, menor tiempo de espera y tratamiento inmediato, entre otros otros. Existiría una baja relación entre atención médica y percepción del paciente.

\section{OBJETIVOS}

General: Caracterizar la atención que se da en el Servicio de Urgencia del Hospital de Llay Llay y determinar la relación entre la percepción de urgencia del usuario y la atención médica recibida.

\section{Específicos:}

- Determinar el promedio consulta/día, consulta/ persona/año, consultas/día según mes del año, consultas/día según día de la semana que se otorgan en el Servicio Urgencia.

- Determinar la preferencia del paciente por el Servicio de Urgencia o Consultorio.

Determinar los motivos por los cuales se prefiere un servicio determinado.

Relacionar la percepción de urgencia del usuario y la atención médica recibida.

- Determinar el porcentaje de pacientes frecuentes versus pacientes infrecuentes.

Determinar el porcentaje de pacientes policonsultantes.

\section{MATERIAL Y MÉTODO}

Estudio descriptivo de las características de la población usuaria del Servicio de Urgencia del Hospital de Llay Llay. La información se obtuvo de la base de datos del Departamento de Estadísticas del Servicio durante el periodo Enero - Diciembre 2003.

Se realizó una encuesta estructurada a una muestra de usuarios de los Servicios de Urgencia y Consultorio entre los días martes 27 y viernes 30 de enero del año 2004.

Estos fueron encuestados en sala de espera del Consultorio o del Servicio de Urgencia del Hospital y debían cumplir con los siguientes criterios de inclusión:

\section{Ser mayor de 15 años.}

Ser paciente o acompañante.

Haber sido atendido, al menos, una vez en el último año en el Servicio de Urgencia del Hospital (excluyendo la atención actual). Haber recibido atención en consultorio. No haber contestado previamente la encuesta.
Se formularon cinco preguntas en formato de múltiple elección en relación al lugar de preferencia de atención, motivos de esta preferencia, percepción de urgencia en la última atención en el Servicio de Urgencia, calificación de la atención médica (urgente o no urgente), $\mathrm{N}^{\circ}$ de consultas al Servicio de Urgencia en el último año y sugerencias.

Los encuestados consignaron sexo y edad.

Los datos obtenidos de las encuestas se procesaron mediante tabulación y análisis de promedios y porcentajes.

Para el análisis, se definieron los siguientes términos operacionales:

1) Percepción Usuaria (PU): Motivo que hace consultar al usuario en el SU. Puede ser:

- Urgente: Aquel usuario que considera su motivo como grave y cree necesitar atención urgente o inmediata (opción A). - No Urgente: Cualquier otro motivo seleccionado $(\mathrm{B}, \mathrm{C}, \mathrm{D}, \mathrm{E})$.

2) Atención Médica (AM): Todo procedimiento que se realizó al usuario durante la última vez que fue al SU. Considerada para fines de este estudio como el Gold Standar. Puede ser:

- Grave: Toda atención que requirió hospitalización u observación por 1 hora o más, independiente de otros procedimientos.

- Leve: Atención que requirió procedimientos como curación de heridas o tratamientos farmacológicos de urgencia.

- No Urgente: Si posterior a la evaluación médica, se le indicó que no correspondía a una urgencia, si sólo se le practicaron exámenes o sólo se derivó al consultorio.

3) Frecuencia de Consulta: Número de veces que el usuario ha acudido al SU en el período de una año. Según el promedio de consultas/ día por usuario en una población determinada, se definen:

- Usuario Infrecuente: Aquel usuario que en un período de un año su número de consultas fue menor al promedio de su población.

- Usuario Frecuente: Aquel usuario que en un período de un año su número de consultas fue mayor o igual al promedio 
de su población.

- Policonsultante: Usuario Frecuente cuya PU es Urgente y su AM es No Urgente.

Para estimar la relación existente entre la Percepción Usuaria y Atención Médica, los resultados fueron analizados en tablas tetracóricas, obteniéndose de estas su Sensibilidad, Especificidad, Valor Predictivo Positivo y Negativo.

\section{RESULTADOS}

En el Servicio de Urgencia del Hospital de
Llay Llay, en el periodo Ene-Dic 2003 se realizó un total de 43.643 consultas médicas*, con un promedio general de 130.66 consultas/día**, con un promedio aproximado de 2 consultas por persona/año.

Las consultas en mayores de 15 años correspondieron al $70,1 \%(30.598)$ de las atenciones (hombres: $38,54 \%$, mujeres: $61,46 \%$ ).

La variación de las consulta promedio/día según mes y día de la semana se pueden observar en las Figuras 1 y 2.

El número de consultas promedio fue de 130,66 consultas al día con rango de 148,8 en agosto a 122,5 en septiembre. Según día de la
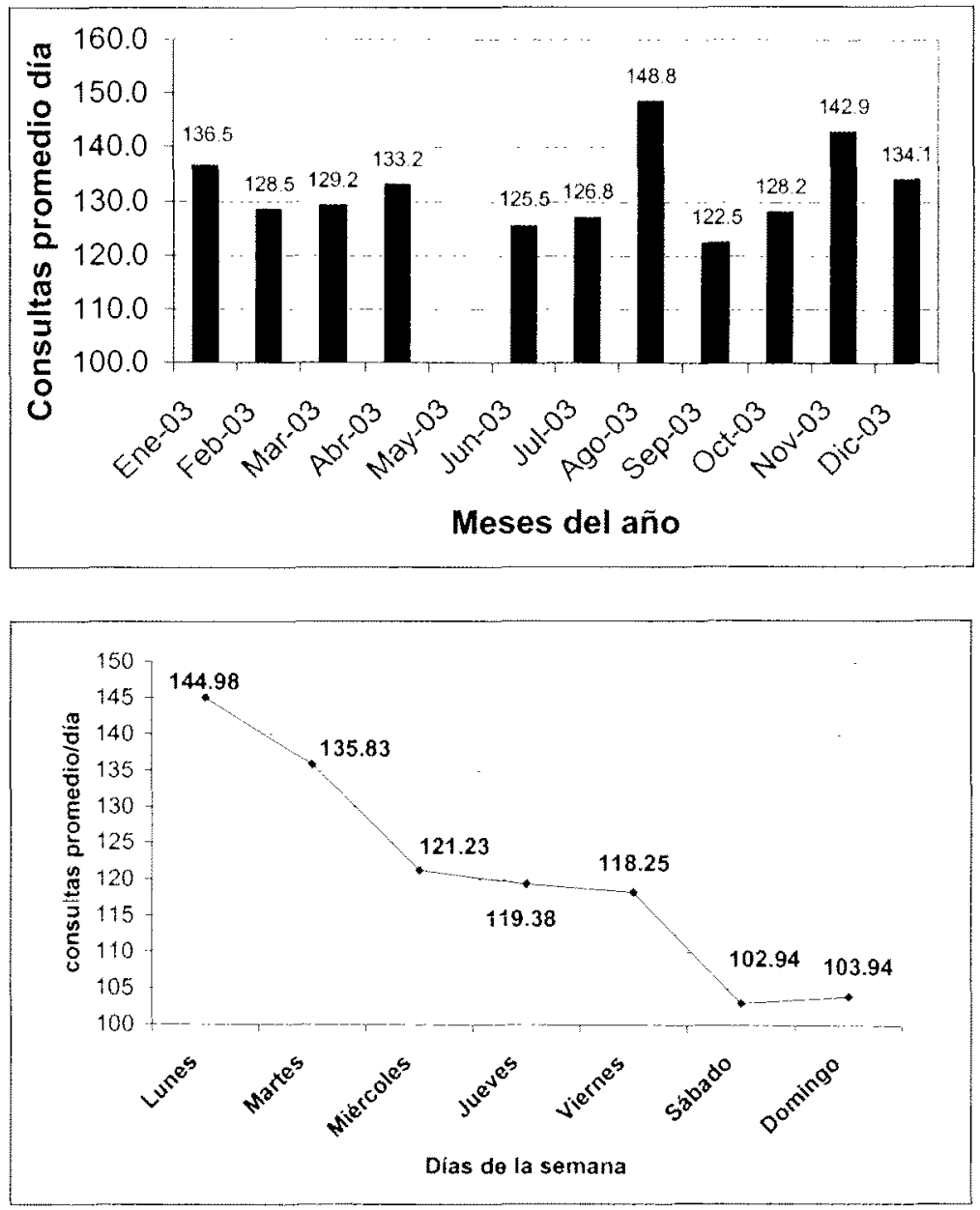

Figura 1. Consultas promedio/día según mes del año.

Figura 2. Curva de consultas promedio/día según dias de la semana.

No se incluyeron las atenciones a embarazadas, dental ni curaciones.

* Se eliminó del número total de días, 31 días correspondientes al mes de mayo 2003 , debido a que los datos no fueron ingresados a la base. 
semana el mayor número de consultas ocurre el lunes, luego declina, observándose el menor número de consulta los fines de semana.

En la encuesta, se solicitó la participación a un total de 111 personas, de las cuales se negaron a contestar 7 ( 2 por ceguera y presbicie, 4 por analfabetismo y 1 por falta de tiempo).

Del total de 104 encuestas se trabajó con 82 $(78,9 \%)$ eliminándose 22 por mal contestadas. De las respondidas: $74,4 \%$ correspondieron a mujeres, y $25,6 \%$ a hombres. El promedio de edad fue de 42,3 (41,2 en hombres y 42,7 en mujeres). El 65\% de las encuestas se realizó en Consultorio y el 34,1 en el Servicio de Urgencia.

Al relacionar lugar de preferencia y lugar de la atención al momento de la encuesta se aprecia que de los encuestados en Consultorio un $25,92 \%$ prefiere la atención de Urgencia y los encuestados en Urgencia prefieren este servicio en un $60,71 \%$ (Tabla 1 ).

Tabla 1. Relación entre lugar de encuesta y preferencia de atención

\begin{tabular}{llccr}
\hline Preferencia & \multicolumn{4}{c}{ Lugar de encuesta } \\
& Consultorio & \multicolumn{2}{c}{ Urgencia } \\
& $\mathbf{n}$ & \% & n & $\%$ \\
\hline Urgencia & 14 & 25,9 & 17 & 60,7 \\
Consultorio & 40 & 74,2 & 11 & 39,3 \\
Total & 54 & 100,0 & 28 & 100,0 \\
\hline
\end{tabular}

Los motivos seleccionados, según el lugar de preferencia se resumen a continuación***

El $69,5 \%$ de los consultantes acudieron al servicio de urgencia por considerar Urgente su problema $(68,9 \%$ en mujeres y 71,4 en hombres). El resto lo hizo por otra motivación (Tabla 2).

Tabla 2. Percepción Usuaria según sexo

\begin{tabular}{lcccccc}
\hline \multirow{3}{*}{ Sexo } & \multicolumn{9}{c}{ Uercepción Usuaria } \\
& $\mathbf{n}$ & $\%$ & $\mathbf{n}$ & $\%$ & n & $\%$ \\
\hline Hombre & 15 & 71,4 & 6 & 28,6 & 21 & 100 \\
Mujer & 42 & 68,9 & 19 & 31,1 & 61 & 100 \\
Total & 57 & 69,5 & 25 & 30,5 & 82 & 100 \\
\hline
\end{tabular}

Entre las opciones No Urgentes que motivaron la última consulta en el Servicio de Urgencia, destacan la falta de disponibilidad de horas en el Consultorio, principal argumento entregado para preferir la atención del Servicio Urgencia $(64 \%)$ tanto por hombres como mujeres. La opción "siempre voy al Servicio de Urgencia" correspondió al $20 \%$ de esta serie, siendo la segunda opción más seleccionada de este grupo. La derivación desde consultorio correspondiente al $12 \%$ de las elecciones y la solicitud de Licencia o Certificado fue manifestado por un $5 \%$.

El 73,2\% tuvo categoría de Urgente $(51,2 \%$ urgente grave y $22 \%$ urgente leve) y $26,8 \%$ atención médica No Urgente (Tabla 3). El porcentaje de pacientes hospitalizados fue de $14,7 \%$.

Al relacionar la Percepción Usuaria, definida como Urgente o no Urgente y la Atención Médica recibida definida como Grave, Leve o No Urgente y combinarla en tablas tetracóricas se obtuvieron los resultados de la Tabla 4.
Urgencia

(A) No debo esperar tanto para que me atiendan

(B) Me dan los medicamentos gratis

(C) Me atienden mucho mejor

(D) No tengo que pedir hora

(E) Resuelven mis problemas rápidamente

(F) Me realizan exámenes de inmediato
$32,25 \%$

$9,67 \%$

$22,58 \%$

$29,03 \%$

$35,48 \%$

$38,70 \%$
Consultorio

$31,37 \%$

$50,98 \%$

$49,02 \%$

$3,92 \%$

$17,03 \%$

$15,68 \%$

*** La suma total no corresponde a un 100\%, ya que se permitió seleccionar hasta dos opciones. 
La discriminación del Usuario entre Urgente (Grave o Leve) y No Urgente (Tabla 4) tiene: Sensibilidad:

$78,3 \%$

Especificidad

$54,5 \%$

Valor Predictivo Positivo:

$82,4 \%$

Valor Predictivo Negativo

$48,0 \%$

Falsos Positivos:

$17,5 \%$

Falsos Negativos

$52,0 \%$

Entre Grave y No Urgente (Tabla 5) tiene: Sensibilidad:

Especificidad

Valor Predictivo Positivo

$78,6 \%$

Valor Predictivo Negativo:

$54,5 \%$

$76,7 \%$

$57,1 \%$

Falsos Positivos:

$23,2 \%$

$42,8 \%$

Entre Leve y No Urgente (Tabla 6) tiene:

Sensibilidad

Especificidad

Valor Predictivo Positivo

Valor Predictivo Negativo

Falsos Positivos

Falsos Negativos

$77,8 \%$

$54,5 \%$

$58,3 \%$

$75,0 \%$

$41,7 \%$

$25,0 \%$

Respecto a la frecuencia de uso del servicio de urgencia se encontró un $48,8 \%$ de usuarios infrecuentes y $51,2 \%$ de usuarios frecuentes.

Los Policonsultantes constituyeron el $6,1 \%$ de los encuestados y su promedio de edad fue de 28 años ( $\pm 9,9$ años).

Tabla 3. Atenciones prestadas según categorías

\begin{tabular}{llr}
\hline Atención médica & n & $\%$ \\
\hline Urgente Grave & 42 & 51,2 \\
Urgente Leve & 18 & 22,0 \\
No Urgente & 22 & 26,8 \\
Total & 82 & 100.0 \\
\hline
\end{tabular}

Tabla 4. Capacidad discriminativa de los usuarios entre Urgente y No Urgente

\begin{tabular}{lccc}
\hline $\begin{array}{l}\text { Percepción } \\
\text { usuaria }\end{array}$ & \multicolumn{3}{c}{$\begin{array}{c}\text { Atención médica } \\
\text { Urgente } \\
\text { Grave y Leve) }\end{array}$} \\
& No Urgente & Total \\
\hline Urgente & 47 & 10 & 57 \\
No Urgente & 13 & 12 & 25 \\
Total & 60 & 22 & 82 \\
\hline
\end{tabular}

Tabla 5. Capacidad Discriminativa de los Usuarios entre Grave y No Urgente

\begin{tabular}{lccc}
\hline $\begin{array}{l}\text { Percepción } \\
\text { usuaria }\end{array}$ & \multicolumn{2}{c}{ Atención médica } & \\
Urgente & $\begin{array}{c}\text { No } \\
\text { Grave }\end{array}$ & Total \\
\hline Urgente & \\
No Urgente & 33 & 10 & 43 \\
Total & 9 & 12 & 21 \\
\hline
\end{tabular}

Tabla 6. Capacidad Discriminativa de los Usuarios entre Leve y No Urgente

\begin{tabular}{lccc}
$\begin{array}{l}\text { Percepción } \\
\text { usuaria }\end{array}$ & $\begin{array}{c}\text { Atención médica } \\
\text { Urgente } \\
\text { Leve }\end{array}$ & $\begin{array}{c}\text { No } \\
\text { Urgente }\end{array}$ & Total \\
\hline Urgente & 14 & 10 & 24 \\
No Urgente & 4 & 12 & 16 \\
Total & 18 & 22 & 40 \\
\hline
\end{tabular}

De los encuestados, 34 (10 hombres y 24 mujeres) incluyeron sugerencias, entre ellas destacan: solicitud de mayor número de médicos, menor tiempo de espera en servicio de urgencia y mejor calidad de atención por parte del personal (calidad humana, técnica y comunicacional).

\section{DISCUSIÓN}

La población estudiada presenta niveles de consulta similares en todo el año. En relación a consultas según días de la semana, se observa una evidente preferencia a consultar los lunes disminuyendo progresivamente hasta alcanzar el mínimo los sábado y domingo. Esto hace pensar que los usuarios evalúan su gravedad durante los días de descanso y al persistir las molestias, acuden a los Servicio de Urgencia en busca de ayuda al inicio de la jornada laboral.

De los resultados obtenidos mediante la encuesta, aplicada a la muestra, se desprende que una mayoría corresponde a mujeres. El promedio de edad fue de 42 años sin diferencias entre sexos, concordantes con la realidad nacional.

Se encontró coincidencia en la preferencia de atención y el lugar en donde se contestó la 
encuesta, lo que se explicaría por la tendencia a elegir el lugar de atención independiente de los motivos que lo llevan a consultar.

Entre los motivos por los cuales los usuarios prefieren un servicio determinado, el tiempo de espera no presentó diferencias entre aquéllos que prefirieron Consultorio o Servicio de Urgencia.

Respecto al beneficio de entrega de fármacos, éste sí constituye un factor a favor de la elección por Consultorio. El porcentaje que manifestó preferencia por el Servicio de Urgencia por la entrega de medicamentos $(9,7 \%)$, pudo corresponder a que en este servicio se administran algunos fármacos de urgencia, sin entregárseles los medicamentos de mantención, función dependiente de Farmacia del Consultorio.

La resolución rápida del motivo de consulta, sería un factor considerado por el usuario, lo que sumado al grupo anterior refuerza la idea de la existencia de pacientes que prefieren una resolución práctica y sintomática del motivo de consulta.

La conformidad con la atención recibida es mayor en el consultorio que en el servicio de urgencia.

La solicitud de hora en el Consultorio es un obstáculo y un motivo importante por el cual los usuarios prefieren el Servicio de Urgencia. El $4 \%$ que indicó que no tiene que pedir hora en Consultorio, denota desinformación del sistema, o podría corresponder a grupos de adulto mayor que tienen esa garantía en ciertos servicios.

La posibilidad de realización de exámenes expeditos en el Servicio de Urgencia, es un elemento a considerar. Sin embargo, este factor no debería influir en la elección, ya que la solicitud de exámenes se puede realizar en forma urgente, también desde el Consultorio. Pero, en este último caso, los usuarios son enviados al Servicio de Urgencia, lo que los llevaría a pensar que sólo en este Servicio se realizan exámenes en forma inmediata.

Se supone que las personas que consultan al servicio de urgencia acuden a éste por una urgencia. Sin embargo, en nuestro estudio, se demuestra la existencia de usuarios en los cuales influyen otros factores que no tienen relación con la necesidad de atención inmediata. El $28,6 \%$ de los hombres y el $34,4 \%$ de las mujeres acudió al Servicio de Urgencia con una Percepción No Urgente, comparable con la experiencia extranjera ${ }^{12}$.

Entre las opciones No Urgentes destaca la falta de disponibilidad de horas en el Consultorio. Se esperaría entonces, un incremento de las consultas en el Servicio de Urgencia durante el fïn de semana, horario en el cual el Consultorio está cerrado, lo que no corresponde a lo observado. Esto podría ser de importancia al momento de planificar una extensión horaria en Consultorio, ya que no se justificaría durante el fin de semana (período de menor demanda).

La solicitud de Licencia o Certificado como motivo de consulta en Servicio de Urgencia, denotan desinformación, ya que este Servicio de Urgencia no entrega certificados ni licencias.

La utilización del Servicio de Urgencia sólo por preferencia personal, costumbre o policonsulta, es un dilema que debiera ser resuelto.

Al cuantificar las atenciones otorgadas, las atenciones médicas leves son en proporción menos que las atenciones médicas graves, con un $14,7 \%$ de hospitalizaciones, lo que explicaría la existencia de un sesgo por parte del entrevistado, ya que al consultársele por la "última atención recibida", referiría la atención más grave (más fácil de recordar) y no su temporalidad.

Al evaluar la capacidad discriminativa de los usuarios, los resultados obtenidos nos muestran que para una Percepción Urgente, un $70,2 \%$ correspondía efectivamente a una patología considerada urgente y de los que consultan con Percepción No Urgente, sólo a un $30,7 \%$ se les consideró como tales, es decir, el médico realizó procedimientos de tipo urgente incluso en pacientes que confesaron acudir a este servicio por motivos No Urgentes. Esto podría reflejar un alto porcentaje de tratamiento "placebo" o cuya indicación médica se basa en la exigencia del usuario.

La sensibilidad de los usuarios es, como se predijo, alta y similar en todos los subgrupos estudiados (78\%). La especificidad es baja, resultando compatible con la mala capacidad 
discriminativa de los no enfermos de reconocerse como tales. Se podría pensar que el porcentaje de Falsos Negativos, correspondiera a una subestimación por parte del usuario de su propia gravedad, lo que queda reflejado en el $12 \%$ de derivados desde el Consultorio. Esto sumado a la baja especificidad, refuerza la idea de una sobreestimación por parte del médico, de la gravedad del usuario.

Sería esperable, dadas las características de la población, un alto porcentaje de Falsos Positivos, lo que, en líneas generales tampoco es así, bordeando valores de $20 \%$, y alcanzando su máximo en la discriminación entre Urgentes Leves y No Urgentes; esto explicaría también la tendencia de los encuestados a recordar el episodio más grave y no el temporalmente más reciente.

Los pacientes frecuentes corresponden aproximadamente al $50 \%$, lo que es concordante con las estadísticas del servicio.

El porcentaje de $6,1 \%$ de policonsultantes es una cifra concordante con lo percibido por el personal de salud. Se destaca que el promedio de edad de estos usuarios, es bajo respecto del grupo estudiado, aunque dicho número no permite obtener resultados concluyentes.

Debido a que la definición utilizada es inédita, no existen cifras que permitan compararla y relacionarla con este estudio ${ }^{12,13}$.

El porcentaje alto de sugerencias sumado a la baja negativa a contestar la encuesta y a la buena disposición general de los encuestados, permite concluir que existe una fuerte motivación por parte de la población para mejorar el sistema de salud que reciben. Esto permitiría implementar programas participativos, los cuales serían bien recibidos por la población.

\section{CONCLUSIONES}

Existirían patrones de conducta poblacional, predecibles y cuantificables, que permitirían la implementación de programas de mejora de los Servicio de Salud.

La atención médica pareciera estar influida por la Percepción Usuaria del propio paciente, ya que a pesar de acudir a un Servicio de Urgencia por patología no
Urgente, se le atiende como tal.

- En la muestra, los usuarios que acuden al Servicio de Urgencia tienen conductas y preferencias distintas de los que prefieren el consultorio, no correspondiéndose necesariamente, Percepción de Urgencia y Atención Recibida.

La atención otorgada en los Servicios de Urgencia a usuarios que no requieren tal atención, podría incentivar el mal uso de estos Servicios.

Las definiciones utilizadas, permiten reiterar este estudio en otros Servicios con similares características.

Se aconseja desincentivar el uso de los Servicios de Urgencia mediante la mayor información a los usuarios de las ventajas y desventajas de cada uno de los servicios.

Se recomienda la implementación de un sistema Triage.

- Se sugiere implementar medidas específicas y realizar evaluación posterior a ellas.

\section{REFERENCIAS}

1.- ALBAR MARÍN M J, MUÑOZ RUIZ E, GARCÍA BURGUILLOS M. Why are hospital emergencies used? Opinion of nursing professionals. Rev Enferm 2003; 26(10): 59-64.

2.- MARDONES J, ESPINOZA R, CRUZ MENA E et al. Urgencia: calidad y costo-eficacia. Boletín Escuela de Medicina. Pontificia Universidad Católica de Chile 1995; 24: 179-82.

3.- GARCÍA ALVARADO R el al. Urgencias Hospitalarias: Análisis del Diseño y Gestión de Servicios de Emergencia Hospitalaria en Chile.

4.- MINSAL. Guía de Planificación y Diseño de Unidades de Emergencia Hospitalaria, División de Inversiones y Desarrollo del Ministerio de Salud, Santiago, Chile, 1999.

5.- SEMPERE T, PEIRÓ $S$, PILAR PINA $S$ et al. Validez del protocolo de adecuación de urgencias hospitalarias. Rev Esp Salud Publica, Jul./Ago. 1999; 73: 4, p.461-475. ISSN 1135-5727.

6. OTERINO D, PEIRO S, CALVO R, SUTII, P. Accident and emergency department inappropriate utilization. An evaluation with explicit criteria. Gac Sanit 1999; 13(5): 361-70.

7.- TAYLOR C, BENGER JR. Patient satisfaction in emergency medicine. Emerg Med J 2004; 21 (5): 528-32.

8.- BRANDON W R, CHAMBERS R. Reducing emergency department visits among high-using patients. J Fam Pract 2003; 52(8): 637-40.

9.- LAURENCE C, SCHUURMAN L. Excess cost of 
emergency department visits for nonurgent care. Health Affairs 1994; 32: 162-71.

10.- PADGETT D K, BRODSKY B. Psychosocial factors influencing non-urgent use of the emergency room: a review of the literature and recommendations for research and improved service delivery. Soc Sci Med 1992; 35: 1189-97.

11.- GUTTMAN N, ZIMMERMAN D R, NELSON $M S$. The many faces of access: reasons for medically nonurgent emergency department visits. J Health Polit Policy Law 2003; 28(6): $1089-120$.

12.- LUCAS R H, SANFORD SM. An analysis of frequent users of emergency care at an urban university hospital. Ann Emerg Med 1998; 32(5): 563-8.

13.- KNE T, YOUNG R, SPILLANE L. Frequent ED users: patterns of use over time. Am J Emerg Med $1998 ; 16(7): 648-52$.

Usted puede comentar éste y otros artículos publicados en la Revista Chilena de Saluda Pública, enviando un correo electrónico a revistasp@med.uchile.cl 